\title{
La jouissance d'une écriture novatrice. Traduire et éditer Claude Simon en russe
}

\section{Alexey Vishnyakov}

\section{(2) OpenEdition}

1 Journals

Édition électronique

URL : http://journals.openedition.org/ccs/953

DOI : $10.4000 /$ ccs.953

ISSN : 2558-782X

Éditeur :

Presses universitaires de Rennes, Association des lecteurs de Claude Simon

\section{Édition imprimée}

Date de publication : 30 avril 2015

Pagination : 105-128

ISBN : 9782753539990

ISSN : $1774-9425$

\section{Référence électronique}

Alexey Vishnyakov, «La jouissance d'une écriture novatrice. Traduire et éditer Claude Simon en russe ", Cahiers Claude Simon [En ligne], 10 | 2015, mis en ligne le 22 septembre 2017, consulté le 01 mai 2019. URL : http://journals.openedition.org/ccs/953; DOI : 10.4000/ccs.953 


\title{
LA JOUISSANCE D'UNE ÉCRITURE NOVATRICE TRADUIRE ET ÉDITER CLAUDE SIMON EN RUSSE
}

\author{
Alexey VISHNYAKOV \\ Institut d'État des Sciences humaines de la région de Moscou
}

Claude Simon a été déjà traduit en russe plus d'une fois. En 1983, La Route des Flandres est publiée dans la traduction d'Eléna Baboun sous la même couverture que Robbe-Grillet, Butor et Sarraute. Cette assez bonne traduction est reproduite en 2000 dans le volume des Prix Nobel français. Dans cette édition, est publié également le Discours de Stockholm traduit par Galina Beliaeva. Les deux livres sont accompagnés de la préface et des notices de Leonide Andreev. En 1989, une traduction faite par Natalia Gorbanevskaya de L'Invitation - étonnamment précise pour une journaliste et non une traductrice professionnelle - est publiée dans la revue parisienne des émigrés soviétiques Continent ( ${ }^{\circ} 56,1989$, p. 239-283). En 2003, cette fois en Russie, paraît la traduction de L'Invitation par Catérina Liamina. Cette dernière comporte certaines déformations du texte simonien ${ }^{1}$.

Cette même année, après l'entretien que Claude Simon m'a accordé, j'ai présenté mon projet d'édition à la commission de sélection de la série éditoriale la plus prestigieuse de Russie Les Monuments littéraires. Cette série supervisée par l'Académie des Sciences accueille déjà plus de 600 livres² $^{2}$ dans son

1. L’article est basé sur deux interventions au Séminaire Traduire Claude Simon le $1^{\text {er }}$ juin 2013. Je tiens à remercier A. Duncan, M. Bertrand, A.-Y. Julien et D. Zemmour pour leur aide dans mes recherches, ainsi que Fatima Zenatti de l'Université Paris 8 pour l'organisation de mon voyage. Mon séjour en France et la participation aux travaux du Séminaire de l'ALCS sont devenus possibles grâce à la bourse octroyée par la Fondation Maison des Sciences de l'Homme (FMSH), et je suis reconnaissant à la Direction scientifique de la FMSH Sonia Colpart et Dominique Richard pour leur attention toujours bienveillante.

2. Quelques parutions récentes: Samuel Beckett (2001), Agrippa d'Aubigné (2001), Simone de Beauvoir (2005), Madame de Lafayette (2007), Bussy-Rabutin (2010), Victor Hugo (2012). 
organisation éditoriale, elle ressemble beaucoup à la "Bibliothèque de la Pléiade " avec chronologie, notices et notes, annexes, illustrations sur la vie de l'écrivain, le texte et les étapes essentielles de sa création ${ }^{3}$.

À la différence de la «Pléiade », il s'agit d'une édition concentrée autour d'un texte. La série des Monuments littéraires présente l'œuvre d'un écrivain à travers un seul ouvrage, ce qui n'exclut pas la présence des autres textes en annexes. De ce point de vue, L'Herbe (un « beau livre " comme l'a appelé Simon, en apprenant mon désir de le traduire) apparaît comme un texte-carrefour résumant des expériences précédentes. De notre point de vue, il ne s'agissait pas seulement d'éditer un texte très abouti de Simon, mais de concevoir cette traduction comme un hommage à une écriture véritablement novatrice. Savoir retransmettre ce sentiment au lecteur est primordial. Outre L'Herbe, cette édition comprend, en annexes, L'Invitation dans la nouvelle traduction de Marina Arias-Vihil. L'Herbe (tout comme Histoire, Les Géorgiques ou L'Acacia) permet de jeter un regard global sur l'évolution protéiforme de la poétique simonienne. L'Invitation et l'interview de Simon de 2003 sont appelés à apporter des couleurs complémentaires à ce tableau.

\section{POUR UNE TRADUCTION « PHILOLOGIQUE »}

Il s'est donc agi, à partir de ces deux textes, de montrer toute la cosmogonie simonienne. Il a fallu d'abord se confronter aux autres difficultés, dont la plus évidente était le choix d'un modèle de traduction. Je voyais deux types de traductions possibles: la traduction "traditionnelle " et la traduction "philologique". La première, où le traducteur, tel le maître-serviteur des Belles infidèles, fait semblant de s'effacer derrière le texte original, est largement répandue. À travers les traductions de ce type, une culture s'approprie les richesses de la littérature étrangère en les faisant siennes. C'est à peu près la démarche de Voltaire traduisant le monologue de Hamlet, de Mérimée traduisant La Dame de pique ou de Dostoïevski traduisant Eugénie Grandet. On peut reprocher à ces traductions le fait que le texte perd, à cause de cette assimilation, son caractère étranger et étrange et une partie importante de son originalité. D'un outil (ou d'un champ) de recherches, il tend à devenir un objet de consommation à signification unique.

Mais c'est précisément à partir de L'Herbe que Simon pose au traducteur ce dilemme qui le fait hésiter entre la perception de son texte comme un objet clos,

3. Voir notre dossier de réception «Simon au pays des soviets: Notes sur la réception de l'œuvre simonienne en Russie ", Cahiers Claude Simon, n9, PUR, p. 145-171. 
déterminé et celle d'une praxis jamais finie et se produisant à tous les niveaux textuels. La phrase, le mot, le phonème même, deviennent des nouds de significations réfutant une seule variante de traduction au profit de l'excroissance des interprétations. La traduction philologique avec sa pluralité d'approches se révèle alors possible et représente une défaite assez honorable.

L'auteur de cette traduction refuse l'ambition de transmuer un texte de nature étrangère en un phénomène enraciné dans sa propre culture. Cette traduction ne cache pas ses côtés problématiques, voit et tente de montrer les subtilités du texte lui-même engendrées par l'esthétique et la philosophie de toute l'œuvre de Claude Simon ${ }^{4}$. Elle souligne ce sens tremblé si cher à Simon dans le texte même ou à travers des notes. D'où les nouvelles fonctions esthétiques de ces dernières qui s'ajoutent aux inévitables explications comme, par exemple, celles qui concernent le globe avec la couronne de mariée (p. 4) que Louise dit avoir cherché des yeux dans la chambre de Marie 5 ou le sudarium, ici mis pour sudatorium (p. 124). Les notes entrent - avec le texte original et le lecteur de la traduction en polylogue et même parfois en discussion (dans mon cas, c'était le plus souvent à propos de L'Invitation).

Pour les textes de L'Herbe et de L'Invitation, j'ai pu constituer environ 300 et 200 notes respectivement, ce qui montre l'importance de ce genre de paratexte dans la forme de l'édition choisie. Dans la mesure où il est question, pour moi, de créer un modèle fonctionnel de la cosmogonie simonienne, je quitte parfois le terrain des enseignements biographiques ou socioculturels, de la linguistique ou de la stylistique, et les notes deviennent des microtextes.

Dans Les Monuments littéraires le texte suit immédiatement la page de titre, tout le reste est relégué en deuxième position, à la fin du volume, ce qui permet une lecture immédiate, d'un seul élan, sans intermédiaires. En même temps, toute lecture des textes de ce niveau de complexité doit aller de pair avec la relecture exégétique basée sur les notes, notices et sur les propres réflexions du lecteur. Ce dédoublement de la démarche de lecture dans le cas du texte traduit est encore un défi de taille pour le traducteur et l'éditeur.

En pleine conscience des pertes au niveau stylistique (et surtout - poétique) dues au changement de la langue, je me suis permis d'intervenir quelquefois

4. Un seul exemple tiré de la traduction, plutôt réussie, de La Route des Flandres: la tournure, primordiale pour la bonne compréhension du monde simonien «Et il me semblait y être, voir cela " (E I, p. 204) E. Baboun traduit fidèlement par rapport aux sens des mots français mais en défigurant le sens, dynamique et producteur, du roman de Simon: "Et j'ai le sentiment de me retrouver de nouveau là-bas, de voir tout cela "

5. Toutes les citations sont empruntées aux éditions des textes de Simon dans la "Bibliothèque de la Pléiade " (EI, pour La Route des Flandres et $E$ II, pour L'Herbe). 
directement dans le texte simonien, ce qui est aussi expliqué dans les notes. Manière, selon moi, de respecter la conception de la métaphore propre à Simon qui la considère comme un moyen de transport. Ainsi, en traduisant les deux passages suivants, je transpose le texte simonien en faisant des renvois aux célèbres poèmes de Pasternak et de Mandelstam (ill. 1, ill. 2) qui sont absents, bien sûr, chez Simon mais faciles à reconnaître pour un amateur de poésie russe, ce qui doit élever le degré lyrique de la traduction sans défigurer sa nature.

Cette option suppose des solutions discutables et parfois risquées dans la quête du transport métaphorique. Mon aventure la plus périlleuse est liée à un passage lyrique et pathétique en forme d'alinéa ( $E I I$, p. 77). Les exemples atteignant ce degré emphatique sont rares chez le Simon d'après L'Herbe, et même ici, ils ne sont déjà que trois (les deux autres figurent à la page 35 et 56), mais de ces trois occurrences, ce petit poème en prose est le plus fascinant. Pour le mettre en valeur, il m'a semblé intéressant de le transcrire en forme de vers libres (ill.3), ou même de versets bibliques. Le résultat m'a laissé perplexe à cause de son évidence trop ostentatoire, et donc contraire au caractère plutôt centripète de l'Ars Poetica de Simon.

Ce bref essai a tout de même été l'occasion de sentir l'affinité élective de la poésie (et de la poétique) simonienne avec celle de Joseph Brodsky, ses énumérations parataxiques à mi-chemin entre la poésie et la prose. D'où la décision d'omettre la présentation strophique originelle de ma traduction en soulignant la trame poétique de ce texte à travers la prosodie (à la façon d'un Guillaume Apollinaire, ou de Simon lui-même et de sa manière des années 60), les choix syntaxiques, stylistiques et lexicaux (soulignés dans la traduction), et même en ajoutant parfois des détails d'ordre poétique (mis en italique dans la traduction), ce qui m'a permis de mettre en valeur la complexité de la composition, l'élan épique et le charme lyrique de ce fragment poétique ${ }^{6}$.

\section{QUELQUES QUESTIONS GÉNÉRALES, CONCLUSIONS INTERMÉDIAIRES ET PROBLÈMES CONCRETS}

Et maintenant quelques observations d'ordre général sur l'expérience de la traduction, la mienne aussi bien que celle des autres traducteurs russes de l'œuvre simonienne.

6. Et pourtant, dans la note prévue pour ce passage, je propose à un lecteur curieux de recherches formelles de reproduire pendant sa relecture/reécriture mon cheminement à tâtons. 
L'Herbe a une particularité de composition: si le dernier tiers du roman dans sa complexité polyphonique ne le cède en rien à La Route des Flandres, dans l'incipit, il n'y a rien de particulièrement difficile. Presque tous les premiers nouveaux romans (L'Emploi du temps de Butor, Le Planétarium de Sarraute, La Jalousie de Robbe-Grillet, Le Fiston de Pinget, La Mise en scène d'Ollier, Moderato Cantabile de Duras) sont composés sur un même schéma, évoquant un jeu électronique: intriguer le lecteur en compliquant les règles lorsqu'il commence à s'orienter. Dans L'Herbe aussi, les difficultés se multiplient et augmentent peu à peu, et cela rend le travail du traducteur plus facile.

Mais il y a les difficultés de traduction de Simon en russe qui sont générées par des causes assez éloignées des particularités de l'écriture simonienne ou de la grammaire française, et cette question est digne d'être traitée avec plus de détails. La liste des traductions en russe est encore trop courte pour faire des généralisations hâtives, mais on peut en tirer quelques conclusions. La traduction de $\mathrm{La}$ Route des Flandres est assez réussie, mais la traductrice trahit parfois le texte simonien. Il lui arrive de remplacer le présent par l'imparfait, ce qui anéantit l'effet de simultanéité, ou de remplacer le participe présent par le présent ou le "passé simple », ce qui affaiblit ou fait disparaître l'effet onirique de la description simonienne que G. Roubichou a très justement appelé l' "évocation ».

De ce point de vue est très caractéristique ce qui s'est passé avec la variation simonienne sur le thème du Verrou de J.-H. Fragonard (ou plutôt inspirée par son dynamisme multidirectionnel) (EI, p. 250-251): dans ce passage, E. Baboun remplace tous les participes par les verbes au présent ce qui change la peinture de Simon en... La Défaite de Reichenfels de Robbe-Grillet! La même transmutation est effectuée avec un "passé simple» - p. 217, sur 10 participes présents sont remplacés par les formes perfectives au passé. Le "contenu " est traduit mais l'air de la poésie simonienne s'est évaporé. Encore plus intéressant est ce qui est advenu à la description de la mort de Wack (célèbre " film privé de sa bande de son ", p. 302, illustration 4-a) où la traductrice, de 10 participes présents, en a repris seulement deux en remplaçant les autres par les verbes au "passé simple " et à l'" imparfait "; sans le vouloir, elle a changé le flux colloïdal simonien en un de ces mêmes "vieux films rayés » avec leur façon de montrer le mouvement de la "façon saccadée, mécanique et précipitée " (L’Herbe, p. 38) qui ont été décrits sarcastiquement par Simon plus d'une fois.

En comparant du point de vue stylistique, la traduction des discours de Georges, de Blum et de Wack (et d'Iglésia), on constate que la plus réussie est la parole de Blum (ce qui est facile à comprendre, si on prend en compte la parenté 
socioculturelle de l'intelligentsia soviétique et de ce personnage déraciné, aigre et voyant partout un prétexte à humiliation - la sienne ou (plus souvent celle d'autrui). En traduisant les propos de Georges, la traductrice se perd assez souvent devant la liberté absolue de l'écrivain dans le maniement, non seulement des thèmes, mais des registres stylistiques. Souvent, elle se heurte à des difficultés insurmontables en traduisant des oxymores paradoxaux de Georges mais surtout ses jurons ou blasphèmes ${ }^{7}$. Et elle ne conserve pas toute la saveur rêche de la langue de Wack et des paysans, par laquelle le lecteur simonien (mais pas le lecteur russe, hélas) accède à un des mondes les plus clos, les plus secrets qui soit, proche de celui de Tristes Tropiques de Lévi-Strauss, qui a hanté Simon durant toute sa vie. Les calembours paronomastiques ou polysémiques sont le plus souvent perdus, parmi ceux-ci - La Saumure " (p. 199), " mort au fond du fossé dévoré par les fourmis » (p. 373) et " les pissenlits " (p. 374), " la même houri la même haletante hoquetante haquenée » (p. 399)... Il faudrait supprimer les corrections faites dans les passages érotiques et religieux et/ou les restituer tout simplement ${ }^{8}$, et, bien sûr, ajouter des notes et des commentaires ${ }^{9}$ pour ce livre d'une complexité extrême. Donc, on pourrait retraduire ce roman en russe. L'analyse de cette traduction au niveau textuel - se situe dans l'illustration 4-a.

Le cas de L'Invitation est encore plus exemplaire, il existe trois traductions faites par trois traductrices distinctes, et en pleine ignorance des résultats des deux autres, ce qui rend leur examen comparatif, même le plus superficiel, inté-

7. Qui sont omis - ou défigurés, ce qui est parfois pire! - de façon systématique: «spèce de con » (p. 236), « je m’en f... » (p. 236), « qu'est-ce que vous racontez putain comme conneries » (p. 237), « C'con-là » (p. 291), " gonzesses" (p. 318), "C'te bête-là et gailles " (p. 319), "ça va encore chier " (p. 319).

8. Sont entièrement omis les passages érotiques (et avec eux - les dissertations de Simon sur les sujets les plus variés) des pages 325-326, ( femme non pas... fauves toisons »), des pages 372-373 (" touffeur, lappant, tige, seins multiples, moiteur fauve, lapper sa..., moule humide d'où sortaient »), de la page 385 (« ma joue sur... boutonnière de chair »), de la page 405 sur « les épouses japonaises». De 12 emplois du verbe pénétrer qui sont presque toujours accompagnés de connotations sexuelles, aucune n’est gardée. Tout ce qui est lié au membre viril - même le pronom $i l$ - est corrigé ou omis. Fait curieux, la traductrice a laissé quelques détails de l'anatomie féminine, à cause, probablement, de l'impossibilité de sauvegarder sans eux les dernières apparences de la description érotique simonienne. Dans tous ces cas, il est impossible de comprendre s'il s'agit de censure ou d'auto-censure, car à cette époque les moeurs soviétiques évoluaient - lentement mais irrésistiblement - vers une plus grande liberté. Mais le dessin classique en tous temps et pays (" un ovale partagé en deux... ", p. 385) est resté proscrit, aussi bien que tout le passage sur "les deux hiéroglyphes les deux principes » (p. 395-396).

9. La traductrice a mal compris les expressions " une vieille chemise aux coins cornés " (p. 214), " une histoire d'os comptés, dénombrés », "ils ont numéroté mes abattis » (p. 240), elle n’a pas repéré l'allusion antisartrienne sur « une fameuse nausée » (p. 327), et quelques autres. Et même dans la dernière salve du texte simonienne composée de quatre épithètes antéposées (" l'incohérent, nonchalant, impersonnel et destructeur travail du temps ", p. 412), cette mise en relief est perdue à cause du refus de la traductrice de trouver en russe un moyen de souligner ce qui est exprimé par ces antépositions. 
ressant. Nous proposons cette analyse à partir d'une description du torrent tirée de l'incipit de la $5^{\mathrm{e}}$ séquence (illustration 4-b). Du point de vue quantitatif, les trois traductions (aussi bien que celle de E. Baboun) correspondent au volume du texte français mais ne le dépassent pas. Les trois traductrices essayent de respecter les règles de la syntaxe russe, ce qui mène inévitablement non seulement à la "normalisation" (donc, à l'affaiblissement de l'effet imaginé par l'auteur) de la syntaxe simonienne mais à l'alourdissement du texte. À cela il existe des causes objectives que nous allons examiner plus bas, toutefois, il faut dire que M. Arias-Vihil a réussi, à moindre perte, à réconcilier les règles grammaticales avec la logique du texte simonien.

En comparant ces trois traductions au niveau de la transmission du souffle prosodique du texte de L'Invitation, on constate que N. Gorbanevskaya est celle qui y a été la plus fidèle. Cette traductrice a été, selon toute apparence, impressionnée et entraînée par le courant simonien, et les côtés forts, ainsi que faibles, de sa traduction peuvent être expliqués par cela. De façon intuitive, elle a essayé, en suivant l'original, de changer la description traditionnelle en représentation dynamique (la fameuse aventure de l'écriture) où tout se trouve en mouvement et en changement incessants. Mais parfois, sans le remarquer, elle en élève le degré expressif, ce qui ne correspond pas à l'osmose propre à Simon de l'émotionnel et du rationnel, des images hardies et des appréciations cruellement froides et précises.

À l'autre extrémité se trouve la traduction de C. Liamina où toutes les règles de la syntaxe russe sont strictement respectées. Et en plus, la description dynamique est supplantée, remplacée par la narration. Les épithètes sont systématiquement remplacées par des formes verbales, ce qui aboutit à la non moins fameuse écriture de l'aventure. Poussée par les meilleurs motifs, la traductrice n'oublie jamais de corriger les "fautes" de l'original, ce qui amène aux résultats prévisibles. La traduction de M. Arias-Vihil peut être estimée comme le juste milieu. Dans la mesure où elle est philologue et chercheur à l'Institut de la Littérature mondiale (IMLI), elle rend son texte plus souple et capable de réconcilier les élans de tous genres du texte de Simon et les impératifs de la langue et de la tradition littéraire russes. L’effet esthétique général produit en fait néanmoins la traduction la plus proche du texte original.

On peut conclure qu'il y a au moins une leçon à tirer de cette traduction à trois mains: un professionnalisme froid ou une admiration de prosélyte peuvent également avoir des conséquences lourdes sur la quête simonienne. Simon est un auteur qu'il faut aborder avec adoration, humilité et précision, en toute compréhension. 
Examinons maintenant de près quelques problèmes concrets posés par la traduction de L'Herbe.

\section{PROBLÈMES DE LEXIQUES, OMISSIONS ET AJOUTS}

\section{sans doute, donc:}

On peut traduire ces locutions ambiguës de plusieurs façons. Simon emploie sans doute 112 fois, et très souvent, il est impossible de la comprendre d'une manière univoque. Dans ce cas, j'explique cette option dans une note spéciale. Dans le cas de l'exemple 1, j'ajoute - pour soutenir Simon dans son désir de jouer avec le lecteur au niveau stylistique - des éléments de la parole orale (pazвe что). Pendant tout l'incipit, Simon va mener ce jeu subtil et subversif où récit et description s'entremêlent sans cesse. L'auteur y accoutume son lecteur, et le traducteur doit faire de même.

Donc a 82 récurrences, et l'exemple 14 est l'illustration de son emploi canonique et le plus fréquent dans L'Herbe avec la fonction d'une charnière permettant au courant textuel de regagner son tracé après s'être éloigné dans un (des) affluent(s). En même temps, donc sert très souvent pour nuancer non une phrase mais le phrasé du texte simonien, comme c'est le cas dans l'exemple des versets examiné plus haut. On peut voir encore une fonction importante de donc dans l'exemple 28 , où je me suis décidé pour la première fois à le traduire à l'aide d'expressions du langage oral " ну так вот "- pour montrer ces oscillations si fréquentes chez Simon entre la narration et la description, la parole personnifiée et identifiable et le mot de l'auteur - caché, invisible mais omniscient et omniprésent, comme celui de Balzac, bien que d'une autre façon. Dans La Route des Flandres, ce tissage de la matière textuelle deviendra encore plus subtil et inventif, mais déjà ici, ce phénomène est présent sous sa forme transitoire (entre Le Vent et La Route des Flandres) très particulière et digne d'être mise en valeur par le traducteur et le commentateur.

Parfois, j'omets quelque chose ou ne traduis pas à la lettre. Chaque fois, ce choix a une explication. Les exemples 3,8 ont perdu la nuance orale à cause de la nécessité d'ajout de plusieurs mots pour sa transmission, en raison des particularités de la syntaxe russe. Mais dans les cas où c'est possible, je verse toujours un peu de cette oralité même si elle est absente dans l'original. Dans l'exemple 4, à cause de la déclinaison russe, j'ai perdu le changement de la signification de rien: dans le texte ce n'est plus le mot-connecteur de la conversation mais le symbole d'un «vide plein" de la vie de Marie. Donc, j'ajoute une note expliquant cela. 
Les citations 7 et 13 sont l'exemple de la polysémie de alors chez Simon. Ici, dans les deux cas, je traduis par " après cela » en perdant, surtout dans le deuxième cas, une nuance de causalité.

Quant aux ajouts, on peut les classer en deux types: sémantico-grammaticaux et "artistiques ». Les premiers sont ceux qui éclaircissent le sens et permettent d'éviter une ambiguïté non-voulue par l'auteur. Dans ce cas, j'ai imité Simon lui-même qui utilise ce procédé assez souvent (par exemple, "ou (les enfants) pas encore rentrés ", p. 16; " et elle (Mademoiselle) », p. 25) non seulement à cause de sa soif d'exactitude mais surtout pour préserver et mettre en relief le caractère polyphonique de son texte, qui est profondément non-univoque. Donc, ces précisions et rectifications jouent leur petit mais visible rôle dans cette symphonie des échos et des réflexions. Naturellement, j'ai procédé de cette façon seulement dans les situations où la syntaxe russe ne m’a pas laissé le choix. Les ajouts du deuxième type sont beaucoup plus volontaristes et risqués. Dans quelques cas (cinq tout au plus), je me suis permis d'introduire dans le texte simonien quelques petits jeux de mots paronomastiques suscités par les virtualités du contexte russe et le désir de garder pendant la traduction le scintillement paronomastique du phrasé de Simon. Mais chaque fois, je le précise dans une note en expliquant les causes de ce changement et en restituant la variante de Simon.

\section{PONCTUATION, SYNTAXE, STYLISTIQUE}

\section{Parenthèses}

Exemple 5: Le lecteur, surtout si c'est son premier roman de Simon, rencontre pour la première fois les célèbres parenthèses simoniennes. Donc, il fallait ne pas l'effrayer dès le début. Chez Simon ce passage est relativement court, très expressif et tend vers une prosodie presque musicale. Il était très important de rendre en russe cette musicalité hypnotisante de la syntaxe simonienne que sa complexité cache d'abord. Dans ce cas, comme dans plusieurs autres, les problèmes de la ponctuation cessent d'être une question technique pour devenir la trame de la musicalité du texte.

Maintenant, examinons deux textes de l'exemple 18. Même si je n'ai pas dépassé les dimensions du passage français et sauvegardé sa prosodie, on peut constater que la ponctuation du texte en russe est devenue plus compliquée, plus lourde. Il n'est pas rare, hélas, de voir qu'on partage une phrase "difficile " en deux ou même en trois segments, ce qui tue le phrasé simonien au nom de son 
contenu, et je n'ai pas fait cela une seule fois. Il faut dire encore que la syntaxe russe compense souvent l'insuffisance (du point de vue de la clarté française) de la rigueur formelle par la richesse de la ponctuation qui aide à comprendre correctement les cas fréquents de liberté syntaxique. Voilà pourquoi un regard russe n'est pas perturbé par cette excroissance de la ponctuation qui n'est pas rare dans ma traduction, bien que, plus d'une fois, ma variante soit plus simple, plus aérée dans ses approches ponctuationnelles. On peut se rappeler dans ce cas l'anecdote du rajout par Simon et Jérôme Lindon dans le manuscrit de La Route des Flandres du minimum des signes de ponctuation sans lesquels la bonne compréhension du roman aurait été problématique. Moi aussi, j'ai essayé de ne pas passer la frontière entre la musique et le pédantisme tout en gardant le sens du texte.

\section{Points de suspension}

Même si Claude Simon ne confère pas aux points de suspension une importance et une richesse égales à celles qu'ils prennent chez Nathalie Sarraute, il imagine, lui aussi, des emplois inhabituels et souvent subversifs de ce signe de la ponctuation. Dans le cas de l'exemple 11, son emploi est tout à fait normal, mais il peut être plus expressif, surtout dans les courtes répliques que les héros s'assènent l'un l'autre comme des coups de poing. Alors la subversion de ce signe par Simon devient surtout frappante en russe aussi bien qu'en français, comme c'est le cas dans l'exemple 24 tiré de la scène finale des deux côtés de la séparation. Vers la fin du roman, les transpositions fictionnelles et narratives deviennent de plus en plus hardies et précises. L'écrivain a bien préparé son lecteur à ce montage à la Buñuel. Dans cet extrait, les deux premières occurrences des points de suspension (je n'ai gardé que la première) sont tout à fait normales. Mais la troisième qui, au premier regard, indique le prolongement de la scène de jalousie dans l'hôtel permet le passage du monologue de Sabine au récit de Louise qui a lieu dans la salle de bains. Il est très difficile de le sentir sans un commentaire dans la note.

\section{Deux points}

Simon traite ce signe typographique d'une manière aussi subversive que tous les autres; les exemples avec:) ou ) : se répètent plus d'une fois, il y a même le deux-points à la fin de l'alinéa (p. 54). Pour être compris correctement, tous ces cas doivent être expliqués dans une note. Mais le cas le plus intéressant, selon moi, est l'exemple 17, où ce signe est employé non pour amorcer le dialogue sur la base d'un fragment descriptif (comme cela se produisait une ligne plus 
haut où l'exposition précède la conversation), mais au contraire pour faire comprendre au lecteur que la description découle du discours des personnages, ce qui montre l'accroissement de l'un dans et à travers l'autre, sorte d'enchevêtrement dans l'écriture simonienne de dit/pensé et de décrit, ce qu'on pourrait appeler "mysleskaze» (forme hybride de la réflexion "mysl» et du discours, "skaze »). Si des passages de ce type ne sont pas trop difficiles à traduire, ils sont, néanmoins, très importants pour la bonne compréhension de la poétique simonienne, et demandent, eux aussi, un examen critique dans une note.

\section{Anacoluthe}

L'anacoluthe est un phénomène tout à fait possible en français mais interdit en russe. Chez Simon il n'est pas rare, et ce qui rend la traduction des phrases où elle intervient encore plus difficile est qu'elle se combine souvent avec les infinitifs et les participes (et qui plus est, à l'intérieur de phrases extrêmement ramifiées) dont les fonctions se distinguent aussi considérablement en russe qu'en français. Prenons le seul exemple 9 où la tournure impersonnelle est accompagnée du participe présent parlant de Marie. Pour adoucir le choc de l'emploi fautif (selon la grammaire russe) au début même (de la lecture par un lecteur novice) du roman, je mets ici, au lieu du participe présent " прижимающая " - qui est tout à fait possible - le gérondif « прижимая ». Ce changement rend le texte plus étrange, inhabituel, dans la mesure où on glisse de l'écoulement lent du participe présent à la suspension colloïdale du gérondif. En refusant un compromis avec la grammaire russe, je procède en même temps à la transmission plus précise du texte simonien.

\section{Syntaxe aponctuationnelle}

J'appelle ainsi les changements radicaux dans l'attitude de Simon envers non seulement la ponctuation mais également la syntaxe. L'exemple 21 est la première émergence dans le texte de L'Herbe de ce procédé qu'il pratique ici pour la première fois de façon systématique. Ce procédé n'est pas très compliqué à traduire à condition de trouver une bonne prosodie, un bon souffle de la phrase russe qui rendent tous les tournants du sens. 


\section{ÉPITHÈTES, PARTICIPE, GÉRONDIF}

\section{Antéposition de l'épithète}

Une particularité de la syntaxe russe encore capable de créer des problèmes est l'absence de la place fixe de l'épithète qui peut précéder le nom (ce qui est le plus répandu) ou le suivre. Voilà pourquoi les antépositions simoniennes - lyriques, pathétiques, ironiques, cruelles - peuvent se décolorer, se ternir, voire disparaître dans les méandres des périodes traduites en russe ${ }^{10}$. Dans l'exemple 10, j'ai gardé en partie le potentiel de cette antéposition en mettant les épithètes en relief, mais souvent, ce n'a pas été possible parce que les virgules de cette mise en relief se perdent parmi les autres. Dans ce cas, j'ai procédé, comme dans l'exemple 16, en utilisant deux tirets, ce qu'on ne peut faire trop souvent si l'on ne veut pas alourdir le texte.

Dans ce roman, Simon mélange souvent les registres de la langue écrite et du discours très oral, et il n'est pas toujours facile de traduire cette alternance correctement. Je me permets donc parfois dès le début quelques ajouts ou transformations pour préparer le lecteur (ex. 2). Ici j'ajoute "никто и " pour m’approcher de la formule du discours oral russe. Ex. 6: la même chose est faite sans aucun changement. Ex. 22: dans ce roman ne sont pas rares les cas où Simon ignore de façon manifeste la hiérarchie des styles (d'ailleurs, comme tant d'autres canons narratifs et fictionnels) en rendant, à cause de ce mélange de tons, très difficile la traduction adéquate; c'est pourquoi dans ce passage, je transpose la tournure orale "ç'a été » par l'apostrophe directe du lecteur «вы или я » (vous et moi).

Les effets de polysémie et de paronomase, ainsi que les calembours, sont également très délicats à traduire. Examinons d'abord l'exemple 12 où j'ai perdu les effets acoustiques ironiques des bonbons gluants qui changent en boutons dépareillés. Je crois que ce sont précisément des impasses pareilles qui nous incitent à chercher des variantes de traduction dans les richesses de notre propre langue et non chez Simon ou dans Le Grand Robert. Certes, il faut être très prudent et chercher longtemps pour trouver parfois. Et l'exemple 19 est un de ces cas où j'ai pu trouver une solution. Dans ma variante, j'ai gardé le contraste des $\mathrm{R}$ rigides, rêches avec les $\mathrm{L}$ bien que ces derniers n'aient plus leur caractère mouillé, et j'ai perdu tout de même en quantité.

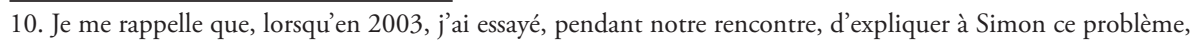
sur son visage vif une expression presqu'enfantine de dépit est apparue, et il m’a dit - comme, probablement, à tant d'autres avant moi: Ah, ça c'est votre problème, pas le mien! 
Le passage 20 est l'exemple de l'homonymie où un texte (dans lequel les images successives s'entrelacent) naît du jeu basé sur la signification plurielle du verbe se rétracter. Donc, je garde les deux significations sous la forme de deux verbes différents.

La traduction, déjà citée, du passage 19 peut servir aussi d'exemple de paronomase plutôt réussie, et pour les calembours, qu'on nous permette d'examiner l'exemple 25. Recouvrant peut être le participe présent du verbe recouvrer, aussi bien que de recouvrir. En penchant vers la deuxième hypothèse, je précise dans la note que pour la juste compréhension du texte simonien, fondamentalement ambigu, il faut aussi avoir en vue la première.

Examinons maintenant le problème du participe présent auquel j'ajouterais celui du gérondif, car souvent chez Simon il est difficile de les distinguer. L'exemple 15 correspond à l'une des premières rencontres de cet usage par le lecteur avec cette particularité de l'écriture simonienne qui est l'emploi massif de ces formes. Le lecteur français est frappé par leur abondance, et le lecteur russe - encore et surtout - par le trou là où il attend, attend mais finalement ne trouve pas le prédicat de l'énoncé. Dans cet exemple, Louise esquisse quelques actions mais aucun de ces gestes n'aboutit. On peut adoucir cet effet de choc à l'aide des participes présents qui, d'après leur structure, sont proches des épithètes et s'accrochent aux noms plus "naturellement " qu'aux verbes - en masquant ainsi l'absence ou la pénurie de ces derniers. C'est ce que j'ai fait ici dans cinq cas sur neuf. Mais dans quatre cas, j'ai employé un gérondif qui accompagne normalement un verbe. Trois gérondifs sont recevables car ils ont leur verbe mais le premier se rapportant à Louise n'a pas de prédicat! Et c'est déjà le commencement de l'élaboration de la structure porteuse de l'évocation simonienne. Non seulement, je n'essaie pas d'adoucir ce processus mais je propose dans la note au lecteur de remplacer tous les participes présents dans ce fragment par des gérondifs. Et cela, pour faire sentir toute l'originalité d'un univers de Simon.

Dans l'exemple 23, je renonce, pour la première fois définitivement à la pratique d'un adoucissement normalisant dans la traduction des périodes simoniennes. Ce passage apparemment cruel dans son objectivité soulignée est l'un des plus remarquables de ce livre. Et comme souvent chez Simon, l'intensité substantielle est accompagnée en contrepoint par des recherches formelles. C'est pourquoi, en suivant le texte français, je rejette toutes les tentatives de (dé)terminer l'action qui est changée de façon définitive (deux ans avant La Route des Flandres!) en état purement simonien: onirique, fantasmatique et cosmogonique. 
Ainsi, j’entraîne le lecteur dans ce que les formalistes russes si chers à Simon ont appelé остраненние, la "découverte ", à l'intérieur de ce qui est normal et bien réglé, du bizarre, de l'étrange à travers lequel percent le vrai et le nouveau. Le lecteur devient un apprenti dans l'atelier de Claude Simon où le chaos et la bizarrerie cachent un élan vers un univers où tout n'est qu'ordre et beauté.

\section{ILLUSTRATIONS}

1. SIMON / BORIS PASTERNAK:

un confus entrecroisement des

branches de pruniers retombantes

(E II, p. 123) нечёткого скрещенья

ветвей понурившихся

La Nuit d'hiver (des Vers de Youri Jivago dans Docteur Jivago):

Sur le plafond couraient des ombres

De pieds, de mains,

Qui se croisaient dans la pénombre, Tels nos destins.
На озаренный потолок

Ложились тени,

Скрещенья рук, скрещенья ног,

Судьбы скрещенья.

\section{SIMON /OSSIP MANDELSTAM:}

le ciel devenu semblable à une plaque de verre devant laquelle ou plutôt sur laquelle semblaient peintes les petites feuilles

(EII, p. 136). стеклянную твердь, перед которой, а точнее - на поверхности которой, был выведен узор. Les expressions soulignées renvoient au poème de 1909 Sur l'émail pâle et bleu (1909). 


\begin{tabular}{|c|c|}
\hline $\begin{array}{l}\text { Donc: la vieille femme - } \\
\text { le vieux, le fragile amas d'ossements, } \\
\text { de peau, d'organes exténués, } \\
\text { aspirant au repos, au néant originel, } \\
\text { gisant - soulevant à peine le drap - } \\
\text { au sein, au centre de la maison, } \\
\text { régnant, } \\
\text { invisible et omniprésente, non } \\
\text { seulement sur toutes les pièces } \\
\text { (présidant - sans qu'il soit besoin de } \\
\text { nul bénédicité - } \\
\text { au repas, à la rupture en commun du } \\
\text { pain } \\
\text { dans le familier tintement des couverts } \\
\text { heurtant les assiettes, } \\
\text { au jacassement absurde de l'autre } \\
\text { vieille femme), } \\
\text { mais encore les débordant, étendant } \\
\text { sa présence, son royaume } \\
\text { au-delà des murs, au-delà même du } \\
\text { râle, } \\
\text { comme si celui-ci n'avait même pas } \\
\text { besoin d'être perçu par l'oreille } \\
\text { pour être entendu jusqu'au bas de la } \\
\text { colline, } \\
\text { et même plus loin, maintenant, dans la } \\
\text { nuit silencieuse, } \\
\text { la nocturne paix du jardin, des } \\
\text { frondaisons et des oiseaux endormis, } \\
\text { des insectes dans l'herbe noire, } \\
\text { sous le ciel noir à peine plus clair là- } \\
\text { bas, } \\
\text { du côté où s'en est allé tout à l'heure } \\
\text { le crépuscule } \\
\text { comme par une blessure par où se } \\
\text { retire lentement } \\
\text { la lumière (p. 77). }\end{array}$ &  \\
\hline
\end{tabular}




\section{FRAGMENTS DES TRADUCTIONS DE LA ROUTE DES FLANDRES ET DE L'INVITATION}

En italique - ce qui n'est pas suffisamment exact ou est fautif, souligné l'omission d'un ou de quelques mot(s), et en gras - une variante particulièrement réussie.

Ill.4-a

\begin{tabular}{|c|c|}
\hline $\begin{array}{l}\text { La Route des Flandres, deuxième } \\
\text { partie, E I, p. 303, } 1235 \text { signes }\end{array}$ & $\begin{array}{l}\text { Traduction par Eléna Baboun, 1983, } \\
\text { c.462-463, } 1330 \text { signes }\end{array}$ \\
\hline $\begin{array}{l}\text { [...] comme s'il continuait à } \\
\text { chevaucher quelque Pégase invisible } \\
\text { qui d'une ruade l'eût fait basculer en } \\
\text { avant exécutant donc au ralenti et } \\
\text { pour ainsi dire sur place une sorte de } \\
\text { double saut périlleux me le montrant } \\
\text { bientôt la tête en bas la bouche } \\
\text { toujours ouverte sur le même cri (ou } \\
\text { conseil qu'il avait essayé de me faire } \\
\text { entendre) silencieux puis couché dans } \\
\text { les airs sur le dos comme un type } \\
\text { étendu dans un hamac et qui laisse } \\
\text { pendre ses jambes à droite et à gauche } \\
\text { puis de nouveau la tête en haut le } \\
\text { corps vertical les jambes commençant } \\
\text { à abandonner la position de celles } \\
\text { d'un cavalier pour se rassembler } \\
\text { pendre parallèlement puis sur le } \\
\text { ventre les bras tendus en avant les } \\
\text { mains ouvertes dans le geste de saisir } \\
\text { d'attraper quelque chose plus loin } \\
\text { comme un de ces acrobates de cirque }\end{array}$ & 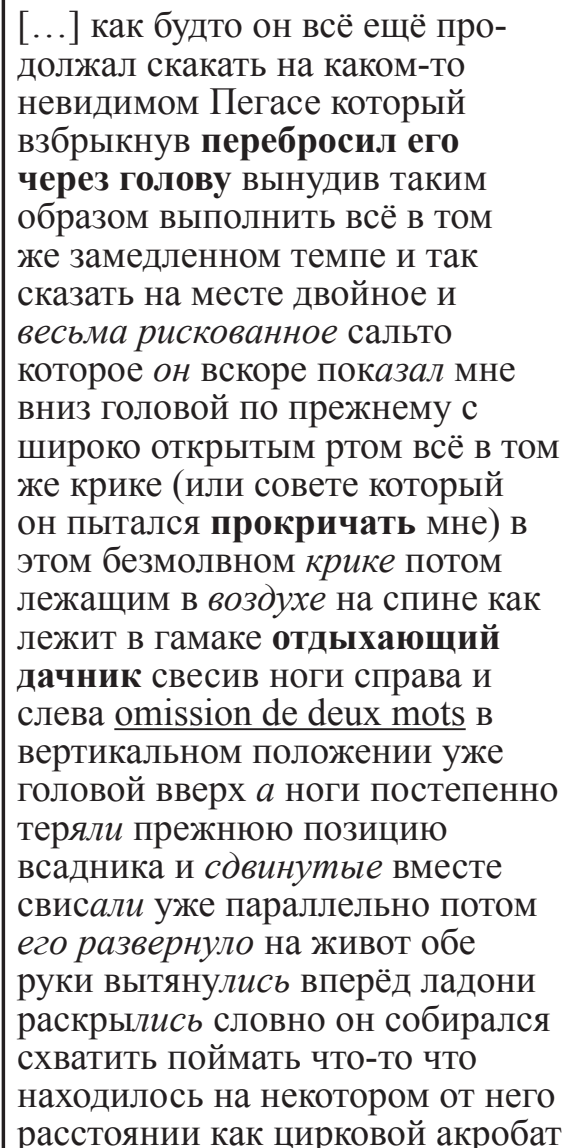 \\
\hline
\end{tabular}




\begin{tabular}{|c|c|}
\hline $\begin{array}{l}\text { [dans l'instant où il se tient rattaché } \\
\text { à rien et délivré de toute pesanteur } \\
\text { entre les deux trapèzes puis à la fin } \\
\text { la tête de nouveau en bas les jambes } \\
\text { désunies et les bras en croix comme } \\
\text { pour me barrer le chemin mais } \\
\text { immobile maintenant plaqué contre } \\
\text { le revers du talus et ne bougeant } \\
\text { plus me regardant le visage empreint } \\
\text { d'une expression surprise et imbécile } \\
\text { je pensais Pauvre Wack il a toujours } \\
\text { eu l'air d'un idiot mais maintenant } \\
\text { plus que jamais il, [...] (CEI, } \\
\text { p. 303). }\end{array}$ & $\begin{array}{l}\text { в тот миг когда он висит } \\
\text { ни за что не держась между } \\
\text { двумя трапециями и тело его } \\
\text { не подчинено закону земного } \\
\text { притяжения потом в конце концов } \\
\text { голова его снова очутилась внизу } \\
\text { ноги разошлись в стороны а руки } \\
\text { раскинулись крестом будто он } \\
\text { желал преградить мне путь но } \\
\text { теперь полностью неподвижный } \\
\text { влипший в придорожный откос не } \\
\text { шевелясь больше и глядя прямо } \\
\text { на меня на его лице застьло } \\
\text { удивлённо-глупое выражение я } \\
\text { подумал Бедняга Вак всегда-то у } \\
\text { него был идиотский вид но сейчас } \\
\text { ещё более идиотский чем обычно, } \\
\text { отіssion d'un тот [...] }\end{array}$ \\
\hline
\end{tabular}

\section{Ill.4-b}

L'Invitation, l'incipit de la 5-e séquence

\begin{tabular}{|c|c|c|c|}
\hline $\begin{array}{l}\text { E II, } \\
\text { p. } 983-984 . \\
988 \text { signes. } \\
24 \text { signes de } \\
\text { ponctuation. }\end{array}$ & $\begin{array}{l}\text { Natalia } \\
\text { Gorbanevskaya, } \\
1989 \text {, p. } 260 . \\
937 \text { signes. } \\
31 \text { signes de } \\
\text { ponctuation. }\end{array}$ & $\begin{array}{l}\text { Marina } \\
\text { Arias-Vihil, } \\
1988,2014 . \\
821 \text { signes. } \\
28 \text { signes de } \\
\text { ponctuation. }\end{array}$ & $\begin{array}{l}\text { Catérina } \\
\text { Liamina, } \\
2003, \text { p. } 59 . \\
947 \text { signes. } \\
35 \text { signes de } \\
\text { ponctuation. }\end{array}$ \\
\hline $\begin{array}{l}\text { Pendant tout le } \\
\text { temps qu'ils pas- } \\
\text { sèrent au pied de } \\
\text { la formidable mon- } \\
\text { tagne ils ne ces- } \\
\text { sèrent d'entendre le } \\
\text { torrent. }\end{array}$ & $\begin{array}{l}\text { В течение всего } \\
\text { времени, которое } \\
\text { они провели } \\
\text { у подножья } \\
\text { исполинской } \\
\text { горы, они } \\
\text { непрестанно } \\
\text { слышали горную } \\
\text { реку. }\end{array}$ & $\begin{array}{l}\text { Всё время, пока } \\
\text { они находились у } \\
\text { подножия горной } \\
\text { громадьl, они } \\
\text { omission d'un mot } \\
\text { слышали шум } \\
\text { потока. }\end{array}$ & $\begin{array}{l}\text { В продолжение } \\
\text { всего времени, } \\
\text { проведённого } \\
\text { ими у подножия } \\
\text { ужасной } \\
\text { горы, они не } \\
\text { переставали } \\
\text { слышать поток. }\end{array}$ \\
\hline
\end{tabular}




\begin{tabular}{|c|c|c|c|}
\hline $\begin{array}{l}\quad \text { Il était } \\
\text { là, invisible, } \\
\text { caché dans } \\
\text { la dépression } \\
\text { où poussaient } \\
\text { les peupliers, } \\
\text { hors d'atteinte } \\
\text { au-delà de } \\
\text { l'infranchissable } \\
\text { grillage qui } \\
\text { entourait le parc } \\
\text { aux luxueuses } \\
\text { résidences, et } \\
\text { d'après son } \\
\text { bruit, l'incessant } \\
\text { et tumultueux } \\
\text { chuintement, on } \\
\text { pouvait l'ima- } \\
\text { giner, écumeux, } \\
\text { avec ses eaux } \\
\text { rebondissantes } \\
\text { dévalant sans fin, } \\
\text { sorties quelque } \\
\text { part de quelque } \\
\text { fantastique } \\
\text { glacier et } \\
\text { débouchant } \\
\text { enfin dans } \\
\text { la plaine (la } \\
\text { plaine, la steppe } \\
\text { desséchée où il } \\
\text { allait se perdre) }\end{array}$ & $\begin{array}{l}\text { Она была здесь, } \\
\text { невидимая, } \\
\text { скрытая в ущелье, } \\
\text { где росли тополя, } \\
\text { недостижимая, } \\
\text { по ту сторо- } \\
\text { ну высоченной } \\
\text { решётки, которая } \\
\text { окружала парк } \\
\text { с роскошными } \\
\text { дачами, и по её } \\
\text { шуму, неустан- } \\
\text { ному, яростно- } \\
\text { му шипенью, } \\
\text { можно было её } \\
\text { вообразить, } \\
\text { пенистую, с } \\
\text { бурлящей водой, } \\
\text { отіsіоп d'un } \\
\text { тот бесконечно } \\
\text { скатывающуюся, } \\
\text { пробившуюся где- } \\
\text { то из какого-то } \\
\text { фантастического } \\
\text { ледника и наконец } \\
\text { вырывающуюся на } \\
\text { равнину (равнина, } \\
\text { иссушенная степь, } \\
\text { где ей предстоит } \\
\text { исчезнуть) }\end{array}$ & $\begin{array}{l}\text { Он шумел } \\
\text { совсем рядом, } \\
\text { невидимый, } \\
\text { прячась } \\
\text { в низине, } \\
\text { заросшей } \\
\text { тополями, } \\
\text { недосягаемый за } \\
\text { неприступной } \\
\text { оградой, } \\
\text { окружавшей } \\
\text { парк с } \\
\text { роскошными } \\
\text { резиденциями, } \\
\text { и по его шуму, } \\
\text { нескончаемому } \\
\text { бурлению, } \\
\text { шипению можно } \\
\text { было вообразить } \\
\text { его пенящимся, } \\
\text { стремительно } \\
\text { несущимся вниз, } \\
\text { вздымая воды, } \\
\text { истекающие } \\
\text { откуда-то } \\
\text { из отіssiоп } \\
\text { d'uп тот } \\
\text { фантастического } \\
\text { ледника, и } \\
\text { выбирающимся, } \\
\text { наконец, } \\
\text { на равнину } \\
\text { (засушливую } \\
\text { степь, в которой } \\
\text { ему суждено } \\
\text { затеряться), }\end{array}$ & $\begin{array}{l}\text { Он был там, } \\
\text { невидимый, скры- } \\
\text { тый в низине, где } \\
\text { росли тополя, не- } \\
\text { досягаемый из-за } \\
\text { высокой и крепкой } \\
\text { решётки, которой } \\
\text { был обнесён парк } \\
\text { с роскошными } \\
\text { резиденциями, } \\
\text { и, слушая его } \\
\text { шум, немолчный } \\
\text { и живой, можно } \\
\text { было представить, } \\
\text { как он без устали } \\
\text { несёт свои воды } \\
\text { вниз, пенясь и } \\
\text { подпрыгивая, } \\
\text { выходя на свет } \\
\text { неизвестно где, } \\
\text { из какого-нибудь } \\
\text { фантастического } \\
\text { ледника, } \\
\text { и наконец } \\
\text { устремляется на } \\
\text { равнину (равнина, } \\
\text { иссохшая } \\
\text { степь, где ему } \\
\text { предстояло } \\
\text { исчезнуть), }\end{array}$ \\
\hline
\end{tabular}




\begin{tabular}{|c|c|c|c|}
\hline $\begin{array}{l}\text { après avoir } \\
\text { franchi } \\
\text { les gorges } \\
\text { encaissées, } \\
\text { dévalé de } \\
\text { cataracte en } \\
\text { cataracte, } \\
\text { encore furieux, } \\
\text { s'ébrouant, } \\
\text { secouant ses } \\
\text { crinières d'eau, } \\
\text { noir et argent, } \\
\text { reformant } \\
\text { sans fin ses } \\
\text { tresses liquides, } \\
\text { bouillonnant, } \\
\text { comme si de } \\
\text { la vieille et } \\
\text { monstrueuse } \\
\text { montagne } \\
\text { parvenait sans } \\
\text { relâche sous } \\
\text { forme de sourd } \\
\text { mugissement la } \\
\text { voix de quelque } \\
\text { monstre, } \\
\text { quelque oracle } \\
\text { moqueur, } \\
\text { indifférent, } \\
\text { porteur de } \\
\text { quelque secret } \\
\text { sans secret. } \\
\text { ( } E \text { II, p. } 983 \text { - } \\
984) \text {. }\end{array}$ & $\begin{array}{l}\text { после того, как } \\
\text { она преодолела } \\
\text { тесные } \\
\text { горловины, } \\
\text { перекатилась } \\
\text { с порога на } \\
\text { порог, всё } \\
\text { ещё яростная, } \\
\text { отфыркиваясь, } \\
\text { потрясая сво- } \\
\text { ей водяной, } \\
\text { исчерна- } \\
\text { серебряной } \\
\text { гривой, } \\
\text { без конца } \\
\text { переплетая } \\
\text { свои текучие } \\
\text { косы, кипя так, } \\
\text { словно от старой } \\
\text { чудовищной } \\
\text { горы } \\
\text { безостановочно } \\
\text { доносился в виде } \\
\text { глухого мычания } \\
\text { голос какого- } \\
\text { то чудища, } \\
\text { какого-то } \\
\text { издевательского } \\
\text { оракула, } \\
\text { равнодушного, } \\
\text { хранящего } \\
\text { некую тайну без } \\
\text { тайны. }\end{array}$ & 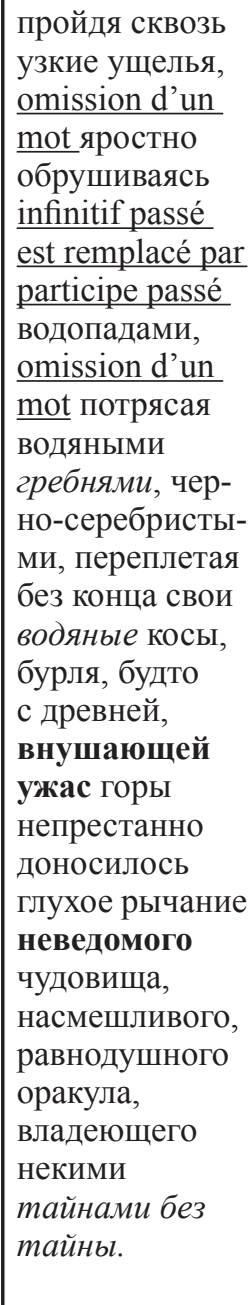 & $\begin{array}{l}\text { преодолев } \\
\text { теснины ущелий, } \\
\text { спрыгнув infinitif } \\
\text { passé est remplacé } \\
\text { par participe passé } \\
\text { с уступа одним, } \\
\text { затем другим } \\
\text { водопадом, } \\
\text { ещё сердитый, } \\
\text { плешущийся, } \\
\text { отіssion d'un mot } \\
\text { встряхивающий } \\
\text { завитками водяной } \\
\text { гривы, чёрно- } \\
\text { серебряный, } \\
\text { без конца } \\
\text { переплетающий } \\
\text { свои текучие } \\
\text { косы, бурлящий, } \\
\text { как если бы из } \\
\text { недр древней и } \\
\text { страшной горы } \\
\text { непрерывно } \\
\text { доносился } \\
\text { глухой рык - } \\
\text { голос какого-то } \\
\text { чудовища, какого- } \\
\text { то насмешливого, } \\
\text { равнодушного } \\
\text { оракула, знаюшего } \\
\text { тайну, в которой } \\
\text { нет тайны. }\end{array}$ \\
\hline
\end{tabular}


5. Exemples sur les problèmes concrets de la traduction de L'Herbe.

\begin{tabular}{|c|c|}
\hline \multicolumn{2}{|c|}{ Les exemples tirés de l'incipit (p. 3-6) } \\
\hline $\begin{array}{l}\text { 1. sinon peut-être son frère, cet } \\
\text { autre vieillard, et sans doute (1) pas } \\
\text { plus qu'elle ne se pleurerait elle- } \\
\text { même }\end{array}$ & $\begin{array}{l}\text { разве что, возможно, брат - ещё } \\
\text { один старик, да и он, наверняка, не } \\
\text { больше, чем она сама себя оплака- } \\
\text { ла бы }\end{array}$ \\
\hline 2. Mais elle ne t'est rien. & - Но ведь она тебе никто и ничто. \\
\hline $\begin{array}{l}\text { 3. ce quelque chose qu'il ne pouvait } \\
\text { pas voir }\end{array}$ & что-то, чего он не мог видеть \\
\hline 4. Rien: elle ne s'est jamais mariée. & $\begin{array}{l}\text { Ничего: она так никогда и не вы- } \\
\text { шла замуж. }\end{array}$ \\
\hline $\begin{array}{l}\text { 5. (à force de réfléchir au meilleur } \\
\text { moyen de porter une robe à peu } \\
\text { près trois fois plus de temps qu'il } \\
\text { n'en faut pour s'user jusqu'à la } \\
\text { trame au tissu dont elle a été } \\
\text { primitivement faite) }\end{array}$ & $\begin{array}{l}\text { (в результате раздумий о } \\
\text { наилучшем способе носки платья, } \\
\text { позволившем использовать его } \\
\text { чуть ли не в три раза дольше, } \\
\text { чем потребуется для того, чтобы } \\
\text { износить ткань, из которой оно } \\
\text { было сшито, до дыр и одних швов) }\end{array}$ \\
\hline $\begin{array}{l}\text { 6. quand nous nous sommes mariés, } \\
\text { Georges et moi }\end{array}$ & когда $\mathrm{Mbl,} \mathrm{Жорж} \mathrm{и} \mathrm{я,} \mathrm{поженились}$ \\
\hline 7. et alors elle a fouillé & и вот тогда она покопалась \\
\hline 8. avec, dessus, une jeune femme & $\begin{array}{l}\text { с изображённой на крышке } \\
\text { молодой женщиной }\end{array}$ \\
\hline $\begin{array}{l}\text { 9. il lui a fallu un moment pour } \\
\text { dérouler, serrant ensuite la boîte } \\
\text { contre elle }\end{array}$ & $\begin{array}{l}\text { для разматывания которой ей } \\
\text { понадобилось некоторое время, } \\
\text { прижимая }\end{array}$ \\
\hline $\begin{array}{l}\text { 10. cet entêtant et sans doute } \\
\text { imaginaire parfum de fraîcheur }\end{array}$ & $\begin{array}{l}\text { дурманящего и конечно просто } \\
\text { померещившегося, иллюзорного } \\
\text { аромата свежести }\end{array}$ \\
\hline $\begin{array}{l}\text { 11. qui maintenant ne tirent plus de } \\
\text { nous qu'un sourire... }\end{array}$ & $\begin{array}{l}\text { стала чем-то вроде воспоминания } \\
\text { о детских страхах, вызывающего } \\
\text { теперь только ульббку... }\end{array}$ \\
\hline $\begin{array}{l}\text { 12. non de bonbons gluants mais de } \\
\text { boutons dépareillés }\end{array}$ & $\begin{array}{l}\text { не в липких конфетах, а в } \\
\text { разрозненных пуговицах }\end{array}$ \\
\hline 13. Et alors, même si & И даже тогда \\
\hline
\end{tabular}




\begin{tabular}{|c|c|}
\hline 14. Pas d'écrin, donc, mais & Итак, никакого футляра, а \\
\hline $\begin{array}{l}\text { 15.la voix s'arrêtant, s'interrompant } \\
\text { brusquement, et Louise restant là, } \\
\text { un peu haletante, comme surprise, } \\
\text { furieuse dravoir tant parlé, regardant } \\
\text { toujours ce quelque chose que } \\
\text { l'autre ne pouvait pas voir [...], et } \\
\text { au bout d'un moment un oiseau } \\
\text { chanta, tout près d'eux, puis, aussi } \\
\text { brusquement, le chant - une } \\
\text { brève série de notes redoublées, } \\
\text { comme une arabesque calligraphiée, } \\
\text { s'enroulant très vite plusieurs fois } \\
\text { sur elle-même dans la répétition de } \\
\text { la même boucle compliquée, puis } \\
\text { s'échappant, sélevant, sétirant [...] } \\
\text { - cessa et, de nouveau, leur parvint } \\
\text { le vacarme lointain et discordant des } \\
\text { moineaux se rassemblant pour la nuit } \\
\text { dans le bosquet de bambous) }\end{array}$ & $\begin{array}{l}\text { (и останавливающийся, } \\
\text { обрывающийся внезапно голос, } \\
\text { и Луиза - замирающая, слегка } \\
\text { задыхающаяся, как бы застигну- } \\
\text { тая врасплох, в досаде на себя за } \\
\text { такое многословие, по-прежнему } \\
\text { раздлядывая это что-то, которое он } \\
\text { не мог видеть [...], и через мгнове- } \\
\text { ние запела какая-то птица, совсем } \\
\text { рядом с ними, затем так же резко } \\
\text { пение - краткая вереница сдвоен- } \\
\text { ных нот, вроде каллиграфической } \\
\text { арабески, многократно и стреми- } \\
\text { тельно свивающаяся в повторении } \\
\text { одного и того же сложного колена, } \\
\text { потом выскальзывая, поднимаясь, } \\
\text { вытягиваясь [...]- прервалось, и } \\
\text { снова до них донеслось отдалённое } \\
\text { и нестройное щебетанье воробьёв, } \\
\text { укладыьвающичся на ночь в } \\
\text { бамбуковой рощице) }\end{array}$ \\
\hline \multicolumn{2}{|c|}{ Autres exemples } \\
\hline $\begin{array}{l}\text { 16.cachés da } \\
\text { obscurité sou } \\
\text { immobiles ( }\end{array}$ & $\begin{array}{l}\text { прячущихся в темноте - густой } \\
\text { и зелёной - под неподвижными } \\
\text { ветвями }\end{array}$ \\
\hline $\begin{array}{l}\text { 17. Donc elle ne partit pas, }[\ldots] \\
\text { disant: "Qu'est-ce que j'irais donc } \\
\text { faire? [...]" : elles se tiennent toutes } \\
\text { deux, (p. 25) }\end{array}$ & $\begin{array}{l}\text { Итак, она больше не ездила туда, } \\
\text { категорически отказывалась ехать, } \\
\text { [...]говоря: «Да и что мне там } \\
\text { делать? /.../»: они вдвоём сидят }\end{array}$ \\
\hline
\end{tabular}




\begin{tabular}{|c|c|}
\hline $\begin{array}{l}\text { 18. le train de sept heures } \\
\text { débouchant de derrière la colline, } \\
\text { ponctuel lui aussi comme le chat, } \\
\text { faisant gronder le pont de fer, } \\
\text { puis disparaissant [...], le bruit } \\
\text { disparaissant, aussi englouti, tandis } \\
\text { que le frémissement des milliers } \\
\text { de feuilles semblait multiplier le } \\
\text { silence, papillotant, pointillant } \\
\text { la masse des arbres, la lumière se } \\
\text { fractionnant en une infinité de } \\
\text { particules miroitantes présentant } \\
\text { alternativement leurs deux faces } \\
\text { vert et argent, clignotant, (p. 17) } \\
10 \text { virgules }\end{array}$ & $\begin{array}{l}\text { семичасовой поезд, вырывающийся } \\
\text { из-за холма, пунктуальный, как и } \\
\text { кот, с грохотом прокатывающийся } \\
\text { по железному мосту, потом } \\
\text { исчезающий [...], исчезающий, } \\
\text { тоже тонущий где-то шум, в то вре- } \\
\text { мя как дрожанье мириад листьев } \\
\text { множило, казалось, тишину - мер- } \\
\text { цая, испещряя мелкими точками } \\
\text { массу деревьев, и свет, дробящийся } \\
\text { на бессчётное множество отсвер- } \\
\text { кивающих частиц, показывающих } \\
\text { (листья осин) попеременно обе их } \\
\text { стороны - зелёную и серебристую, } \\
\text { помаргивающие; } \\
13 \text { virgules, } 2 \text { tirets, } 1 \text { paire de } \\
\text { parentheses, } 1 \text { point-virgule }\end{array}$ \\
\hline $\begin{array}{l}\text { creux, la forme moulée de son } \\
\text { dans la terre molle (p. 176) } 4 r \text {, }\end{array}$ & $\begin{array}{l}\text { вмятину от своего черепа, плавную } \\
\text { форму для выплавки в пластичном } \\
\text { грунте } 3 p, 3 л\end{array}$ \\
\hline $\begin{array}{l}\text { politique, la nation, } \\
\text { en train de fondre, de se } \\
\text { oute vitesse, exactement } \\
\text { e huître sous les gouttes } \\
\text { une peau de chagrin dont } \\
\text { (p. 21) }\end{array}$ & $\begin{array}{l}\text { её государственная и национальная } \\
\text { целостность как раз в это } \\
\text { время рушилась, с огромной } \\
\text { скоростью отрекалась от себя, } \\
\text { съёживалась - совершенно } \\
\text { так же, как устрица от капель } \\
\text { лимонового сока, как шагреневая } \\
\text { кожа, границы которой, }\end{array}$ \\
\hline 1. "...quarant & $\begin{array}{l}\text { «...сорок лет уже почти сорок лет } \\
\text { прошло с нашей первой встречи } \\
\text { а я вас не понимаю родная сестра } \\
\text { мужчины за которого я вышла } \\
\text { замуж сорок лет назад а я вас не } \\
\text { знаю» }\end{array}$ \\
\hline
\end{tabular}




\begin{tabular}{|c|c|}
\hline $\begin{array}{l}\text { 22. la dernière fois que nous avons } \\
\text { vu cette façon de s'habiller, de se } \\
\text { tenir, ça été par des gens de classes } \\
\text { inférieures qui ne faisaient qu'imiter } \\
\text { avec retard ce quils avaient vu faire } \\
\text { aux riches (p. 37) }\end{array}$ & $\begin{array}{l}\text { подобный фасон одежды, подобные } \\
\text { манеры, мы, вы или я, видели у } \\
\text { представителей низших классов, } \\
\text { которые всего лишь имитирова- } \\
\text { ли с опозданием то, что подсмо- } \\
\text { трели у богатых }\end{array}$ \\
\hline $\begin{array}{l}\text { 23. de la manière suivante: [...] le } \\
\text { domestique revenant de faire des } \\
\text { courses en ville }[. . .] \text {, l'apercevant au } \\
\text { passage, assise sous le marronnier } \\
{[\ldots] \text {; et plus tard Julien ressortant }} \\
\text { pour laver la voiture et la voyant } \\
\text { alors toujours assise à la même } \\
\text { place, et à ce moment un peu } \\
\text { surpris, regardant mieux, trouvant } \\
\text { dans son attitude quelque chose } \\
\text { d'étrange, [...] et à mesure qu'il } \\
\text { s'approchait marchant de plus en } \\
\text { plus vite, puis se mettant à courir, } \\
\text { et, [...] s'immobilisant, la regardant } \\
\text { [...]. (p. 41) }\end{array}$ & $\begin{array}{l}\text { вот как: [...] слуга, } \\
\text { возвращающийся [...] из города, } \\
\text { проезжая мимо, замечая её, } \\
\text { сидящую под каштаном [...]; а } \\
\text { позже Жюльен, снова выходящий, } \\
\text { чтобы помыть машину и тогда } \\
\text { видящий, что она сидит там же, и в } \\
\text { этот момент немного удивившись, } \\
\text { приглядевшись, замечающий в её } \\
\text { позе что-то странное, необычное, } \\
\text { [...] и по мере приближения идя } \\
\text { всё быстрее, потом переходя на бег, } \\
\text { и замерев за метр до неё, глядя на } \\
\text { неё, [...]. }\end{array}$ \\
\hline $\begin{array}{l}\text { 24. pendant trois ans tu m'as } \\
\text { trompée avec elle, crois-tu que } \\
\text { parce que je me suis tue, que } \\
\text { parce que tu ne t'es jamais laissé } \\
\text { surprendre, que j'ai besoin, qu'une } \\
\text { femme a besoin... comme si ces } \\
\text { sortes de choses il était nécessaire } \\
\text { de... ce sont des choses qui se } \\
\text { sentent, qu'une femme... elle le } \\
\text { retrouve à la brèche du mur, je l'ai } \\
\text { vue plusieurs fois descendre vers le } \\
\text { petit bois (p. 108) }\end{array}$ & $\begin{array}{l}\text { три года ты изменял мне с ней, } \\
\text { думаешь, раз я молчала, раз я } \\
\text { ни разу не смогла уличить тебя, } \\
\text { женщине незачем... такие вещи } \\
\text { чувствуются нюхом, и женщина... } \\
\text { она встречается с ним у пролома } \\
\text { стены, я много раз видела, как } \\
\text { она спускается к леску }\end{array}$ \\
\hline $\begin{array}{l}\text { 25.la pluie du Seigneur, recouvrant, } \\
\text { enveloppant lentement la terre } \\
\text { ténébreuse et fertile (p. 133) }\end{array}$ & $\begin{array}{l}\text { дождь Господа Вседержителя, } \\
\text { медленно покрывающий, } \\
\text { обволакивающий мрачную и } \\
\text { плодородную землю }\end{array}$ \\
\hline
\end{tabular}




\begin{tabular}{|l|l|}
\hline 26. donc, les deux sœurs réussissant & ну так вот, на то, что они \\
à élever leur frère, non seulement \\
dans le sens courant du terme, mais \\
dans sa pleine acception, (p. 22)
\end{tabular}$\quad \begin{aligned} & \text { уарабатывали, сёстры ещё } \\
& \text { только в расхожем зната - не } \\
& \text { но в полном его смысле: }\end{aligned}$

